\title{
Towards Reliable Quantification of Steel Alloys at Low Voltage
}

\author{
Philippe T. Pinard ${ }^{1}$, Erkki Heikinheimo ${ }^{2}$, Xavier Llovet $^{3}$ and Silvia Richter ${ }^{1}$ \\ ${ }^{1}$ Central Facility for Electron Microscopy, RWTH Aachen University, Aachen, Germany. \\ ${ }^{2}$ Dept. of Materials Science and Engineering, Aalto University, Espoo, Finland. \\ ${ }^{3}$ CCiTUB, University of Barcelona, ES-08028 Barcelona, Spain
}

Whether it be inclusions, precipitates or segregation at grain boundaries, the development of new steel alloys and processes hinges on the chemical quantification of submicrometer features. The combined requirements for high spatial resolution and accurate measurements of several elements make field emission electron microprobes suitable instruments for studying steel alloys. For quantitative analyses, the spatial resolution is determined by the x-ray emission volume the dimensions of which depend on the sample composition, the beam energy and the overvoltage ratio. For most elements, lowering the beam energy directly translates into the necessity of using lower energy x-ray lines. However, the improvement of the spatial resolution comes with additional challenges, mainly a lower intensity (worst statistics), a larger influence of contamination and oxidation, and inaccuracies of the quantification.

To evaluate the influence of the contamination, Monte Carlo simulations were performed to calculate the apparent decrease of the $\mathrm{Cr}$ concentration due to absorption in the carbon layer. The thicknesses of the latter were determined by measuring the height of contamination spots acquired at different beam energies inside a JEOL JXA-8530F microprobe using an atomic force microscope. The results show smaller contamination at low beam energies, but a non-negligible decrease $(0.4 \mathrm{wt} \%)$ of the $\mathrm{Cr}$ concentration (Fig 1). This illustrates the need of anti-contamination device(s) for low voltage analysis.

The inaccuracies of the quantification, when low energy $\mathrm{x}$-ray lines are used, have been recently highlighted by a round robin on low voltage analysis of steel alloys [1] and by a study of Fe-Si compounds [2]. The proximity between the La lines and their L3 absorption edge has been proposed as the main reason for the inaccuracies due to the large uncertainties in the mass absorption coefficients (MAC). It has been shown by Gopon et al. [2] that using the Fe Ll line (no overlap with absorption edges and less influence by the chemical environment) yields a more accurate quantification of Fe-Si compounds. In this work, the same strategy was used to re-evaluate the round robin and other reference steel samples. Measurements were performed at $5 \mathrm{keV}$ and a liquid nitrogen trap was used. A good agreement with the reference concentrations was found for $\mathrm{Fe}, \mathrm{Cr}$ and $\mathrm{Ni}$ on most samples (Table 1).

Despite the improvement of the accuracy, quantification using the Ll lines has some important limitations. Due to their low intensity, it is not possible to detect minor elements unless the counting time is significantly increased. With an acquisition time of $120 \mathrm{~s}$ per point, it was not possible to detect $\mathrm{Co}$ and $\mathrm{Cu}$ concentrations below $0.5 \mathrm{wt} \%$ in this study. Another limitation is the strong overlap of $\mathrm{Cr}$, $\mathrm{Mn}, \mathrm{Fe}$ and $\mathrm{Co} \mathrm{La}$ and $\mathrm{Ll}$ lines on a layered dispersive element crystal. Interference corrections are not unknown in microprobe analyses, but the interfering lines have usually a lower intensity (e.g. higher order line) than the interfered ones. This is not the case for the Ll lines. The difficulty to accurately correct these large interferences explains the large errors (3-4 wt\%) on the Mn concentration (Table 1).

It is therefore of great interest to further study the La lines inaccuracies. Llovet et al. [1] obtained some improvements when experimentally measured self-absorption MACs [3] were used for Fe and Ni. To continue this investigation, self-absorption and inter-element MACs were measured on P11 (Fig 2). These preliminary results showed no improvement over tabulated MACs (Table 2) which may be explained by the remaining deviations between the experimental and calculated intensities. 
[1] X. Llovet et al, IOP Conf. Series: Materials Science and Engineering 32 (2012), p. 012014.

[2] P. Gopon et al, Microsc. Microanal. 19 (2013), pp. 1698-1708.

[3] J. L. Pouchou and F. M. A. Pichoir, "Microbeam Analysis", San Francisco Press (1988)

[4] D. Drouin et al, Scanning 29 (2007), pp. 92-101.

[5] N. W. M. Ritchie, Microsc. Microanal. 15 (2009), pp. 454-468.

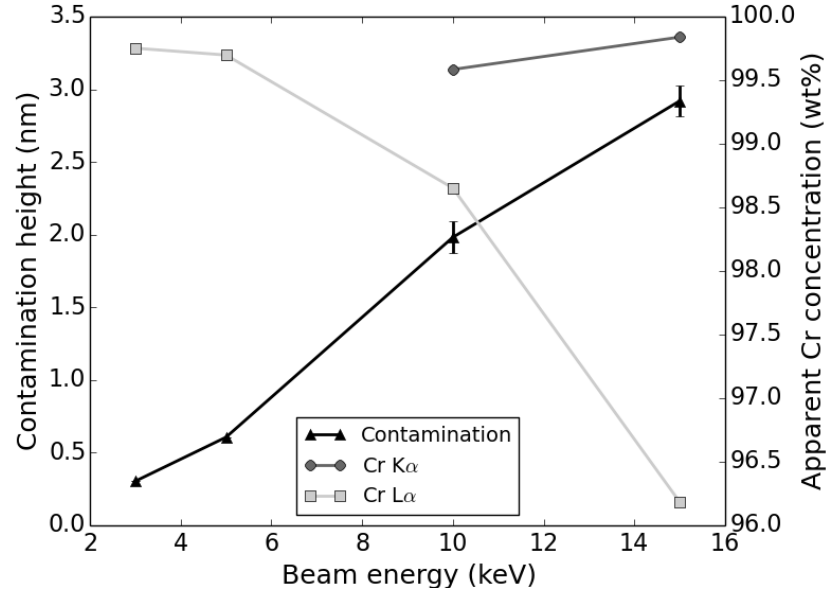

Fig. 1: Height of contamination rings on a GaAs wafer at different beam energies ( $10 \mathrm{nA}, 60 \mathrm{~s}$ dwell time) measured using an atomic force microscope and apparent $\mathrm{Cr}$ concentrations from Monte Carlo simulations (WinCasino [4]) of a carbon layer on a $\mathrm{Cr}$ substrate.

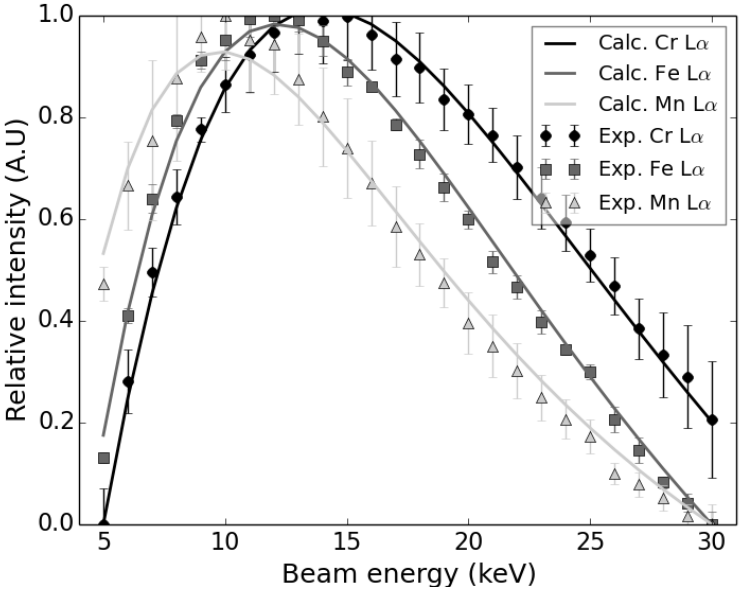

Fig. 2: Variation of the $\mathrm{Cr}, \mathrm{Fe}$ and $\mathrm{Ni} \mathrm{L} \alpha$ experimental (net) and calculated intensities as a function of the beam energy on sample P11. Experimental intensities were acquired for

$120 \mathrm{~s}$ on 4 positions. Intensities were calculated using DTSA-II (XPP). Method in [3] was used to fit intensities.

\begin{tabular}{|c|c|c|c|c|c|c|c|c|c|}
\hline & $\mathrm{Si}$ & $\mathrm{Cr}$ & $\mathrm{Mn}$ & $\mathrm{Fe}$ & $\mathrm{Co}$ & $\mathrm{Ni}$ & $\mathrm{Cu}$ & Mo & Total \\
\hline$A C X$-P07 (ref) & 0.32 & 18.35 & 1.38 & 70.61 & 0.12 & 8.13 & 0.43 & 0.29 & \\
\hline ACX-P07 & 0.33 & 18.64 & 5.85 & 70.80 & 0.0 & 7.47 & 0.0 & 0.33 & 103.43 \\
\hline$A C X$-P11 (ref) & 0.48 & 17.18 & 1.48 & 66.51 & 0.34 & 11.24 & 0.31 & 2.16 & \\
\hline ACX-P11 & 0.51 & 17.63 & 5.71 & 66.43 & 0.0 & 11.44 & 0.12 & 2.11 & 103.95 \\
\hline$A C X$-P13 (ref) & 1.34 & 24.98 & 1.61 & 50.57 & 0.05 & 20.02 & 0.75 & 0.40 & \\
\hline ACX-P13 & 1.01 & 24.85 & 5.97 & 49.76 & 0.0 & 18.87 & 0.12 & 0.40 & 101.38 \\
\hline$A C X$-P15 (ref) & 0.58 & 28.34 & 0.74 & 25.72 & 0.03 & 39.75 & 1.54 & 3.11 & \\
\hline ACX-P15 & 0.50 & 27.21 & 6.51 & 19.57 & 0.0 & 39.77 & 0.0 & 3.19 & 96.75 \\
\hline ACX-260 (ref) & 0.38 & 16.64 & 1.60 & 67.34 & 0.13 & 10.52 & 0.34 & 2.83 & \\
\hline ACX-260 & 0.38 & 16.76 & 5.33 & 66.70 & 0.0 & 10.10 & 0.0 & 3.12 & 102.28 \\
\hline
\end{tabular}

Table 1: Quantitative results using the Ll lines (in wt\%). Quantification with DTSA-II (XPP, FFAST MACs).

\begin{tabular}{|c|c|c|c|c|c|c|c|c|c|}
\hline & $\mathrm{Si}$ & $\mathrm{Cr}$ & $\mathrm{Mn}$ & $\mathrm{Fe}$ & $\mathrm{Co}$ & $\mathrm{Ni}$ & $\mathrm{Cu}$ & $\mathrm{Mo}$ & Total \\
\hline$A C X-P 11(\mathrm{ref})$ & 0.48 & 17.18 & 1.48 & 66.51 & 0.34 & 11.24 & 0.31 & 2.16 & \\
\hline Tabulated & 0.48 & 16.92 & 4.87 & 75.24 & 0.41 & 14.90 & 0.93 & 2.17 & 115.92 \\
\hline Experimental & 0.47 & 19.43 & 6.36 & 77.16 & 0.45 & 12.91 & 0.93 & 2.16 & 119.87 \\
\hline
\end{tabular}

Table 2: Comparison of the La quantitative results using tabulated (FFAST) and experimental MACs 